\title{
Ocean Ambient Noise Studies for Shallow and Deep Water Environments
}

\author{
Martin Siderius and Lanfranco Muzi \\ Portland State University \\ Electrical and Computer Engineering Department \\ $1900 \mathrm{SW} 4^{\text {th }}$ Ave. \\ Portland, OR 97201 \\ Phone:(503) 725-3223 fax:(503) 725-3807 Email: siderius@pdx.edu \\ Award Number: N00014-12-1-0017 \\ http://www.ece.pdx.edu/Faculty/Siderius.php
}

\section{LONG-TERM GOALS}

The objective of this research is to study the ocean ambient noise field by means of new physics-based processing techniques, to determine ways to exploit noise for environmental characterization and to improve sonar-system performance.

\section{OBJECTIVES}

Effective operation of active and passive sonar systems relies on accurate modeling of sound propagation in the environment of the target and receiver. In shallow littoral water, propagation is affected by interaction with the acoustic waveguide boundaries, i.e. the sea surface and the seabed. The seabed reflection loss in particular is a primary contribution to the transmission loss, and is included in shallow-water propagation models as a power reflection loss coefficient, a function of frequency and grazing angle.

A simple passive technique for estimating the bottom loss by beamforming the ambient-noise field using a vertical line array has been developed by Harrison and Simons [Harrison, 2002]. The advantages of passive bottom-survey techniques include simpler measurement requirements, decreased risk of counter-detection, and minimal environmental impact.

Harrison and Simons' technique has so far been applied to data collected by moored or drifting arrays of lengths of the order of several to tens of meters. If this technique could be implemented using much shorter arrays, one could envision a cost-effective bottom-survey system composed by an array mounted on an Autonomous Underwater Vehicle (AUVs). Combining ambient-noise-based bottom survey with the versatility and simplicity of AUVs would result in a system capable of covering extended areas without requiring controlling surface vessels, complex equipment or human interaction during the mission.

The possibility of AUV deployment has made rigid, short arrays increasingly attractive, but their poor angular resolution represents a significant drawback for the purpose of bottom-loss estimation. However, beamforming has some inherent limitations, which affect in particular the angular resolution: All other array parameters being equal, the angular resolution improves when the array length 


\section{Report Documentation Page}

Form Approved

OMB No. 0704-0188

Public reporting burden for the collection of information is estimated to average 1 hour per response, including the time for reviewing instructions, searching existing data sources, gathering and maintaining the data needed, and completing and reviewing the collection of information. Send comments regarding this burden estimate or any other aspect of this collection of information,

including suggestions for reducing this burden, to Washington Headquarters Services, Directorate for Information Operations and Reports, 1215 Jefferson Davis Highway, Suite 1204, Arlington

VA 22202-4302. Respondents should be aware that notwithstanding any other provision of law, no person shall be subject to a penalty for failing to comply with a collection of information if it

does not display a currently valid OMB control number.

1. REPORT DATE

30 SEP 2014

4. TITLE AND SUBTITLE

Ocean Ambient Noise Studies for Shallow and Deep Water Environments

6. AUTHOR(S)

7. PERFORMING ORGANIZATION NAME(S) AND ADDRESS(ES)

Portland State University,Department of Electrical and Computer Engineering,1900 SW 4th Ave,Portland,OR,97207

9. SPONSORING/MONITORING AGENCY NAME(S) AND ADDRESS(ES)

12. DISTRIBUTION/AVAILABILITY STATEMENT

Approved for public release; distribution unlimited

13. SUPPLEMENTARY NOTES

14. ABSTRACT

15. SUBJECT TERMS

16. SECURITY CLASSIFICATION OF:

a. REPORT

unclassified b. ABSTRACT

unclassified c. THIS PAGE

unclassified
17. LIMITATION OF ABSTRACT

Report (SAR)
Same as
3. DATES COVERED

00-00-2014 to 00-00-2014

5a. CONTRACT NUMBER

5b. GRANT NUMBER

5c. PROGRAM ELEMENT NUMBER

5d. PROJECT NUMBER

5e. TASK NUMBER

5f. WORK UNIT NUMBER

8. PERFORMING ORGANIZATION REPORT NUMBER

10. SPONSOR/MONITOR'S ACRONYM(S)

11. SPONSOR/MONITOR'S REPORT NUMBER(S) 
increases [Johnson 1993]. The research described in this document focuses on estimating the reflection loss from the bottom by array processing of marine ambient noise, with a special focus on investigating and expanding the potential and capabilities of short arrays for this application.

Our recent efforts have focused on processing techniques that, by improving the performance of short arrays in reflection-loss estimation, can support the design of the AUV-based bottom-survey system described above. In recent work, we have proposed a new derivation in frequency-wavenumber domain of the power reflection coefficient from the array spatial coherence function [Publications \#1 and \#2]. Besides providing further theoretical support for Harrison and Simons' technique, whose original justification was based on an energy-flux argument, this also led to a processing technique that exploits the physical properties of the noise field to improve the angular resolution of the estimated reflection loss. The work has been finalized this year with a journal publication [Publication \#3].

Furthermore, we have proposed and investigated a new technique for improving the reflection-loss estimation performance of very short arrays. We first studied its application in simulation and existing datasets. Later, our collaboration with the NATO-STO Centre for Maritime Research and Experimentation (CMRE, La Spezia, Italy) resulted in our participation to the REP14-MED measurement campaign (June 2014), during which ambient-noise data were collected both from a conventional vertical line array, and from an array designed as a prototype for a future AUV-mounted system. We then conducted the analysis of the application of the new technique to this original acoustic dataset.

\section{APPROACH}

A theoretical expression for the un-normalized spatial coherence function between two hydrophones in a vertical-line array is given by Harrison [Harrison, 1996]:

$$
\begin{aligned}
C_{\omega}(z)=\int_{0}^{\pi / 2} \frac{2 \pi \sin \theta_{s} \cos \theta_{r}}{1-R_{s}\left(\theta_{s}\right) R\left(\theta_{b}\right) e^{-a s_{c}\left(\theta_{r}\right)}} \\
\cdot\left\{e^{i(\omega / c) z \sin \theta_{r}} e^{-a s_{p}}+R\left(\theta_{b}\right) e^{-i(\omega / c) z \sin \theta_{r}} e^{-a\left[s_{c}\left(\theta_{r}\right)-s_{p}\left(\theta_{r}\right)\right]}\right\} d \theta_{\mathrm{r}} .
\end{aligned}
$$

In Eq.(1), $C_{\omega}(z)$ is the coherence function for the hydrophone pair, assumed to be aligned with the $z$ axis, with the first hydrophone at $z=0$. Furthermore $\theta_{r}, \theta_{s}$, and $\theta_{b}$ are the ray angles at the receiver, the surface, and the bottom; $s_{c}$ and $s_{p}$ are the complete and partial ray-path lengths, whose dependence on $\theta_{r}$ is determined by the sound-speed profile in the water column; $\omega$ is the angular frequency; $c$ is the sound speed at the receiver in the medium, and $R$ and $R_{s}$ are the bottom and surface power reflection coefficients. For the sake of simplicity, the dependence of the reflection coefficients on frequency is not indicated explicitly. Note that $a$ is the power attenuation per unit length.

Although $R$ depends on the frequency of the signal, one could make the often correct hypothesis that the amplitude terms in Eq. (1) are less important than the exponentials - in other words, although the reflection loss does depend (sometimes dramatically) on the frequency, we hypothesize that the spatial coherence function is essentially frequency independent. When this assumption is made, the 
dependence of the coherence function on the sensor spacing $z$ lies primarily in the two exponentials, where $z$ always appears multiplied by the angular frequency $\omega$. This means that multiplying $z$ by an integer factor (as is done to obtain the coherence function between different hydrophone pairs in the array) has the same effect as leaving $z$ unaltered and multiplying $\omega$ by the same factor. The preceding statement can be exploited to extrapolate the coherence function measured by an $M$-element array: The maximum spacing for which the coherence function can be measured from data is $z=(M-1) d$, and the (extrapolated) value of the function at $z=n d(n \geq M)$ can be obtained by assuming:

$C_{\omega}(n d) \approx C_{n \omega /(M-1)}[(M-1) d]$.

This methodology (hereafter referred to as "Frequency-based array reconstruction", or FBR) is applicable as long as there are data available at the higher frequency required in Eq.(2), which depends on the sampling frequency and design of the acquisition system. The choice of the maximum spacing in Eq.(2) is made to maximize the sensor spacing that can be synthetically reconstructed with a given upper frequency limit.

\section{WORK COMPLETED}

A new technique (FBR) for improving the performance of very short arrays in reflection-loss estimation has been theorized. After a first confirmation from OASN [Schmidt, 2004] simulations of the validity of the assumption at the basis of FBR, the technique was applied to measured data from available acoustics datasets collected in the past by CMRE. Both Martin Siderius and Lanfranco Muzi participated in the REP-14 experiment and the techniques described were extended to the new REP14MED dataset.

\section{RESULTS}

The bottom-loss estimates shown in this section are obtained by processing array data acquired during three separate experiments by the NATO-STO Centre for Maritime Research and Experimentation (CMRE - formerly NATO Undersea Research Centre).

TABLE I. Datasets and array basic features - all deployments were drifting, design
\begin{tabular}{ccccc} 
frequency assumed at $c=1500 \mathrm{~m} / \mathrm{s}$. \\
\hline Dataset ID & $\begin{array}{c}\text { Num. of } \\
\text { elements }\end{array}$ & Spacing (m) & $\begin{array}{c}\text { Sampling freq. } \\
\text { (Hz) }\end{array}$ & $\begin{array}{c}\text { Design freq. } \\
\text { (Hz) }\end{array}$ \\
\hline Boundary '03 & 32 & 0.50 & 6000 & 1500 \\
Boundary '03 & 32 & 0.18 & 12000 & 4166 \\
REP14-MED & 32 & 0.18 & 50000 & 4166 \\
\hline \hline
\end{tabular}

In order to test FBR, first the reflection loss has been estimated by Harrison and Simons' technique using data from the full-length array. Then the procedure is repeated using only a subset of array elements, to see the effect of an array of reduced length on the estimated reflection loss. Finally, the data from the same subset of array elements is used to apply FBR, and the result is compared to the two previous estimates. The plots in Figure 1 show the results for data from the Boundary 2004 experiment. Note how the striations visible in the full-array result (due to the presence of layering in the bottom) lose a good amount of details when using only 20 of the available 32 elements. This effect is due to the lower angular resolution of the shorter array. Finally, note how FBR, by affording the 
reconstruction of the contribution to the spatial coherence function of the missing 12 sensors using only data from the same 20 sensors of the subarray (but over a wider frequency range), is capable of recovering most of the detail lost by the shorter array.

The same considerations can be applied to the plots in Figure 2, where the same procedure is applied to data from the Boundary 2003 experiment. The tighter spacing of the array makes it possible to estimate the reflection loss over a wider frequency range (up to the design frequency of $4166 \mathrm{~Hz}$ ). In this case, the relatively low sampling frequency does not allow FBR to reconstruct the reflection loss from the longer array up to the design frequency, but the available frequency range shows that the technique can still recover the detail lost by the shorter array.

Finally, Figure 3 shows the same procedure applied to data from the REP14-MED experiment. In this case, the subarray includes only 8 elements, so that its total length of $1.26 \mathrm{~m}$ is close to that of an AUVdeployable array. The loss of detail in reflection loss, when Harrison and Simons' technique is applied to the subarray is dramatic. In this case the application of FBR is more "extreme" than before, as the attempt is made of quadrupling the length of the subarray, to reach the full length of the original 32element array. Even so, the technique proves capable of recovering a great amount of detail by exploiting the full frequency range of the subarray data.

\section{IMPACT/APPLICATIONS}

This work may have a significant impact on several Navy sonar systems (e.g., ASW, MCM, underwater acoustic communications). Knowing the seabed properties will improve at-sea situational awareness by being able to accurately predict acoustic propagation. And, because this is a passive method it can be designed into a system used for covert activities, low power applications and can be used even in environmentally restricted areas. 

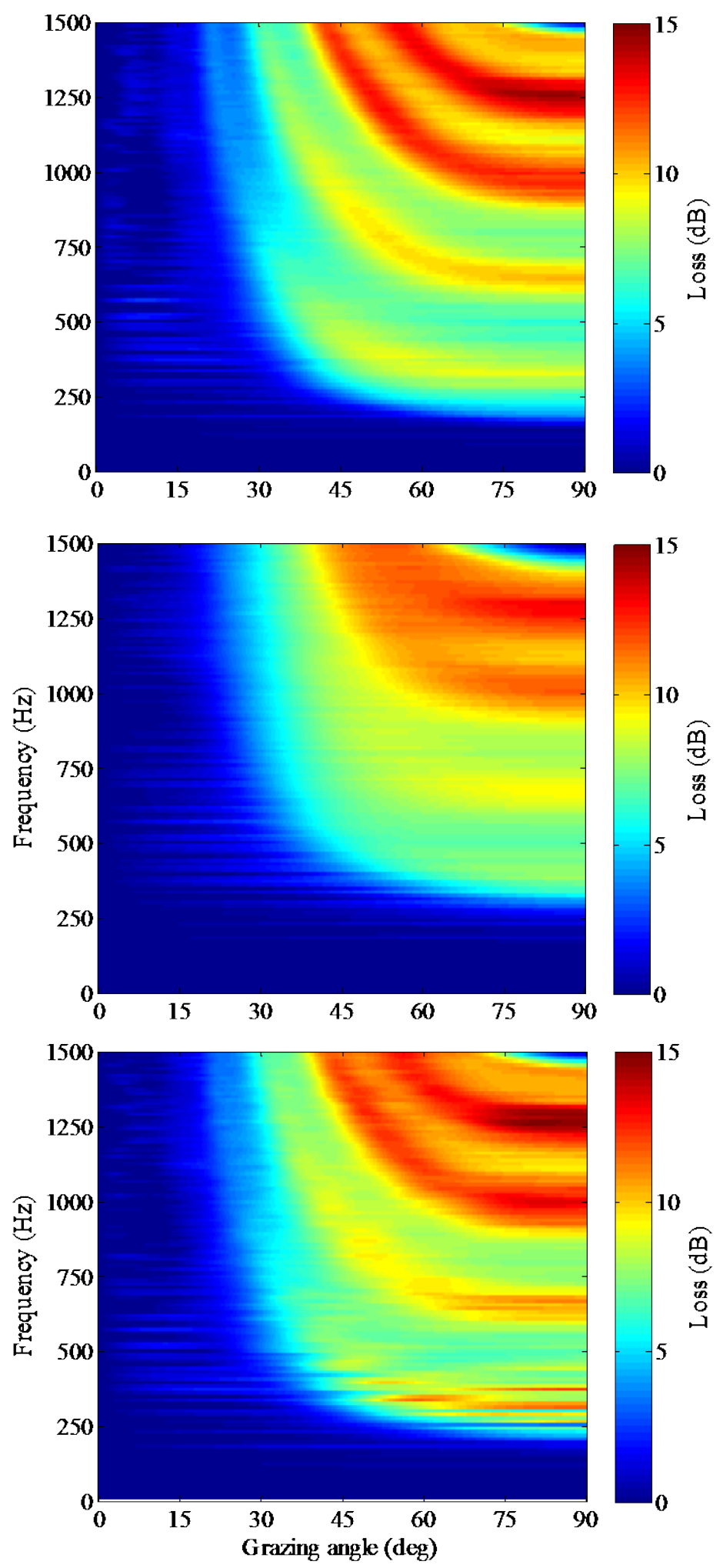

Figure 1: Data from the Boundary 2004 experiment: Reflection loss estimated using data from all the 32 array elements (top), and from only the first 20 elements by the conventional technique (center), and by reconstructing the 12 missing elements by FBR (bottom). 

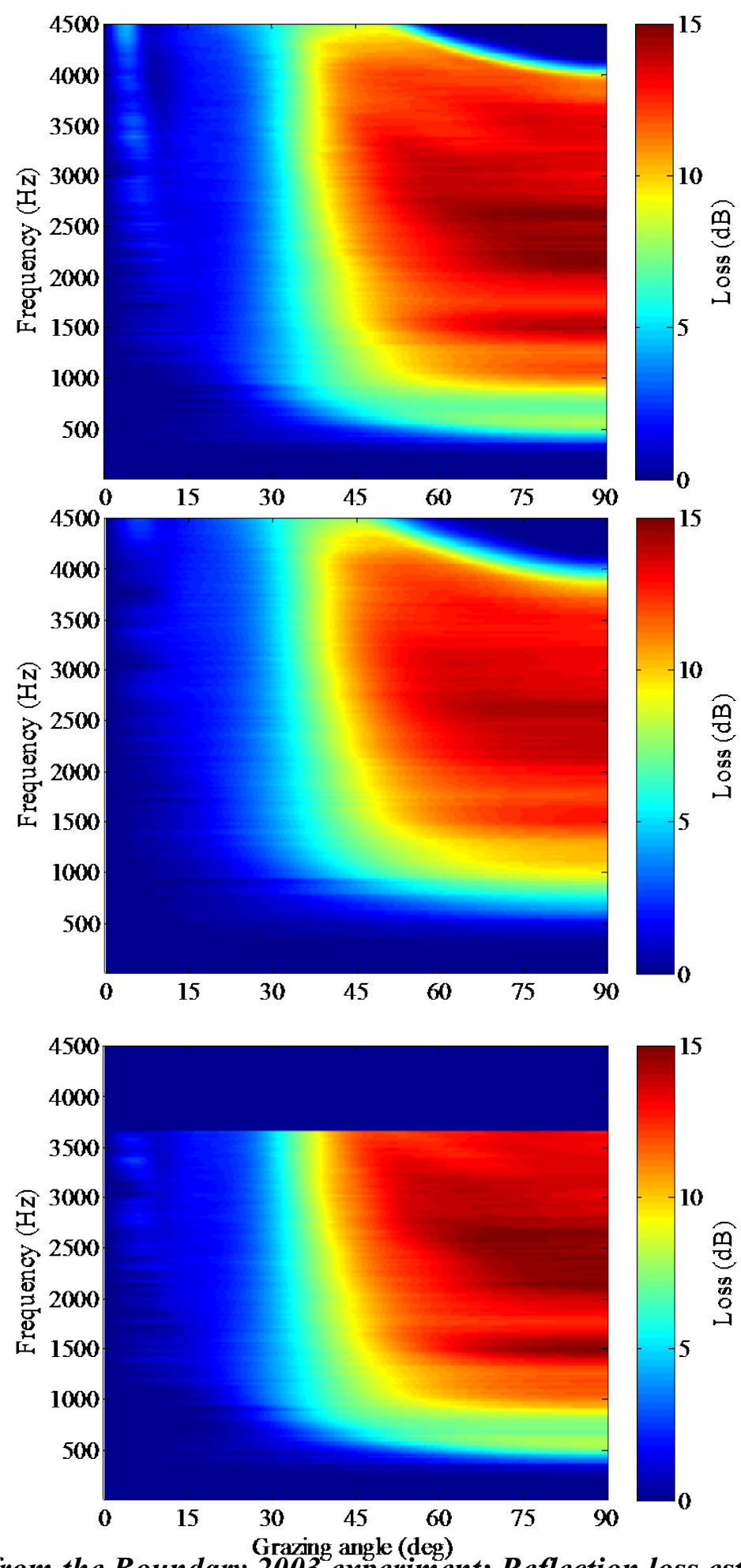

Figure 2: Data from the Boundary 2003 experiment: Reflection loss estimated using data from all the 32 array elements (top), and from only the first 20 elements by the conventional technique (center), and by reconstructing the 12 missing elements by FBR (bottom). Note that, in this case, the frequency range of available data does not allow to reach the array design frequency of $4166 \mathrm{~Hz}$ in the reconstructed array. 


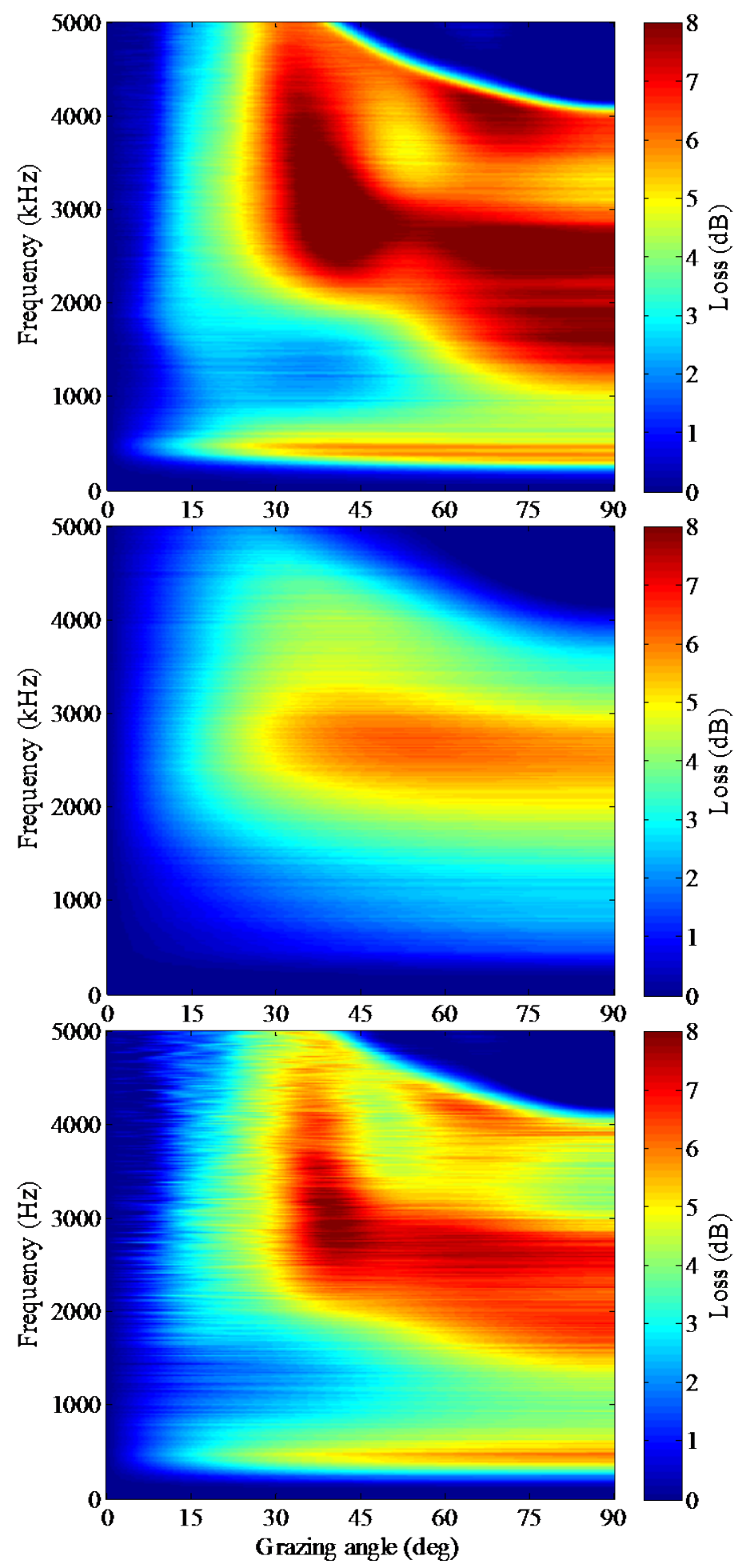

Figure 3: Data from the REP14-MED experiment: Reflection loss estimated using data from all the 32 array elements (top), and from only the first 8 elements by the conventional technique (center), and by reconstructing the 24 missing elements by FBR (bottom). 


\section{REFERENCES}

[Harrison, 2002] C. H. Harrison and D. G. Simons, "Geoacoustic inversion of ambient noise: A simple method," J. Acoust. Soc. Am., 112, 1377-1389 (2002).

[Harrison, 1996] C. H. Harrison, "Formulas for ambient noise level and coherence," J. Acoust. Soc. Am. 99, 2055-2066 (1996).

[Johnson, 1993]D. H. Johnson and D. E. Dudgeon, Array signal processing concepts and techniques, (Prentice-Hall, Upper Saddle River, 1993.

[Schmidt, 2004] H. Schmidt, OASES Version 3.1 User Guide and Reference Manual, Massachusetts Institute of Technology, http://acoustics.mit.edu/faculty/henrik/oases.html, Cambridge, MA, (2004).

\section{PUBLICATIONS}

1. M. Siderius, L. Muzi, C. H. Harrison, and P. L. Nielsen, "Synthetic array processing of ocean ambient noise for higher resolution seabed bottom loss estimation," J. Acoust. Soc. Am., 133, EL149-EL155 (2013). [Accepted, refereed]

2. L. Muzi and M. Siderius, "Synthetic-array beamforming for bottom-loss estimation using marine ambient noise," J. Acoust. Soc. Am., Proceedings on Meetings in Acoustics (POMA) 19, 070033 (2013). [Conference]

3. L. Muzi, M. Siderius, J. Quijano, S. Dosso, "High-resolution bottom-loss estimation using the ambient-noise vertical coherence function,” J. Acoust. Soc. Am. (2014). [Submitted, refereed]

4. L. Muzi and M. Siderius and P. Nielsen, "Experimental studies on passive bottom loss estimation from a compact array mounted on an autonomous underwater vehicle", Proceedings Underwater Acoustics 2014, Rhodes, Greece [Conference]. 